\title{
Cystic fibrosis: terminology and diagnostic algorithms
}

\author{
K De Boeck, M Wilschanski, C Castellani, C Taylor, H Cuppens, J Dodge, \\ M Sinaasappel, on behalf of the Diagnostic Working Group
}

There is great heterogeneity in the clinical manifestations of cystic fibrosis (CF). Some patients may have all the classical manifestations of CF from infancy and have a relatively poor prognosis, while others have much milder or even atypical disease manifestations and still carry mutations on each of the CFTR genes. It is important to distinguish between these categories of patients. The European Diagnostic Working Group proposes the following terminology. Patients are diagnosed with classic or typical $\mathrm{CF}$ if they have one or more phenotypic characteristics and a sweat chloride concentration of $>60 \mathrm{mmol} / \mathrm{l}$. The vast majority of CF patients fall into this category. Usually one established mutation causing CF can be identified on each CFTR gene. Patients with classic CF can have exocrine pancreatic insufficiency or pancreatic sufficiency. The disease can have a severe course with rapid progression of symptoms or a milder course with very little deterioration over time. Patients with non-classic or atypical CF have a CF phenotype in at least one organ system and a normal $(<30 \mathrm{mmol} / \mathrm{l})$ or borderline $(30-60 \mathrm{mmol} / \mathrm{l})$ sweat chloride level. In these patients confirmation of the diagnosis of CF requires detection of one disease causing mutation on each CFTR gene or direct quantification of CFTR dysfunction by nasal potential difference measurement. Non-classic CF includes patients with multiorgan or single organ involvement. Most of these patients have exocrine pancreatic sufficiency and milder lung disease. Algorithms for a structured diagnostic process are proposed.

See end of article for authors' affiliations

Correspondence to: Professor K De Boeck Department of Pediatrics, Pediatric Pulmonology, University Hospital of Leuven, Herestraat 49, 3000 Leuven, Belgium; christiane.deboeck@uz. kuleuven.ac.be

Received 9 March 2005 Accepted

29 November 2006

Published Online First

29 December 2005 ince the discovery of the cystic fibrosis transmembrane conductance regulator - $($ CFTR $)$ gene, it has become obvious that there is great heterogeneity in the clinical manifestations of cystic fibrosis (CF). ${ }^{12}$ Patients may have all the classical manifestations of $\mathrm{CF}$ from infancy and have a relatively poor prognosis. On the other hand, some patients have much milder or even atypical disease manifestations and still carry mutations on each of the CFTR genes. In many of these patients the diagnosis is only made during adult life and the prognosis for survival may be excellent. ${ }^{3}$ It is very important to distinguish these categories of patients and to have a consensus about terminology of these different disease patterns for avoidance of unnecessary and burdensome treatment and incorrect assumptions about prognosis for the individual patient, and for insurance purposes. It is also important for an understanding of the disease outcome in epidemiological and clinical studies. ${ }^{3}$ Physicians need to be aware of the many and varied facets of $\mathrm{CF}$ and should also consider the diagnosis of CF when these rarer presentations occur. An algorithm to arrive at the correct diagnosis in the most efficient way will avoid unnecessary tests and save healthcare resources. If the diagnosis is made in good time, appropriate treatment can be instituted thereby favouring a better outcome. The structured diagnostic process presented in the algorithms in figs 1 and 2 is the product of discussions between experts in the field of CF diagnosis

\section{TERMINOLOGY AND DIAGNOSTIC ALGORITHMS \\ Classic or typical CF}

Patients are diagnosed with classic or typical CF if they have one or more phenotypic characteristics and a sweat chloride concentration of $>60 \mathrm{mmol} / \mathrm{l}$. The phenotypic CF characteristics are chronic sinopulmonary disease, specific or characteristic gastrointestinal or nutritional abnormalities, salt loss syndromes, and male genital abnormalities resulting in obstructive azoöspermia. The majority of CF patients suffer from classic CF. Usually one established CF causing mutation can be identified on each CFTR gene. Patients with classic CF can have exocrine pancreatic insufficiency or pancreatic sufficiency. The disease can have a severe course with rapid progression of symptoms or a milder course with very little deterioration over time.

\section{Non-classic or atypical CF}

Non-classic or atypical CF describes individuals with a CF phenotype in at least one organ system and a normal $(<30 \mathrm{mmol} / \mathrm{l})$ or borderline (30$60 \mathrm{mmol} / \mathrm{l}$ ) sweat chloride level in whom confirmation of the diagnosis of CF requires detection of one disease causing mutation on each CFTR gene or direct quantification of CFTR dysfunction by nasal potential difference measurement. ${ }^{45}$ This includes patients with multiorgan involvement as well as patients with single organ system involvement. Most of these patients have exocrine pancreatic sufficiency and milder lung disease. When two mutations are detected, at least one is by previous experience

Abbreviations: CBAVD, congenital bilateral absence of the vas deferens; $C F$, cystic fibrosis; ICM, intestinal current measurement; IRT, immunoreactive trypsinogen; PD, potential difference 


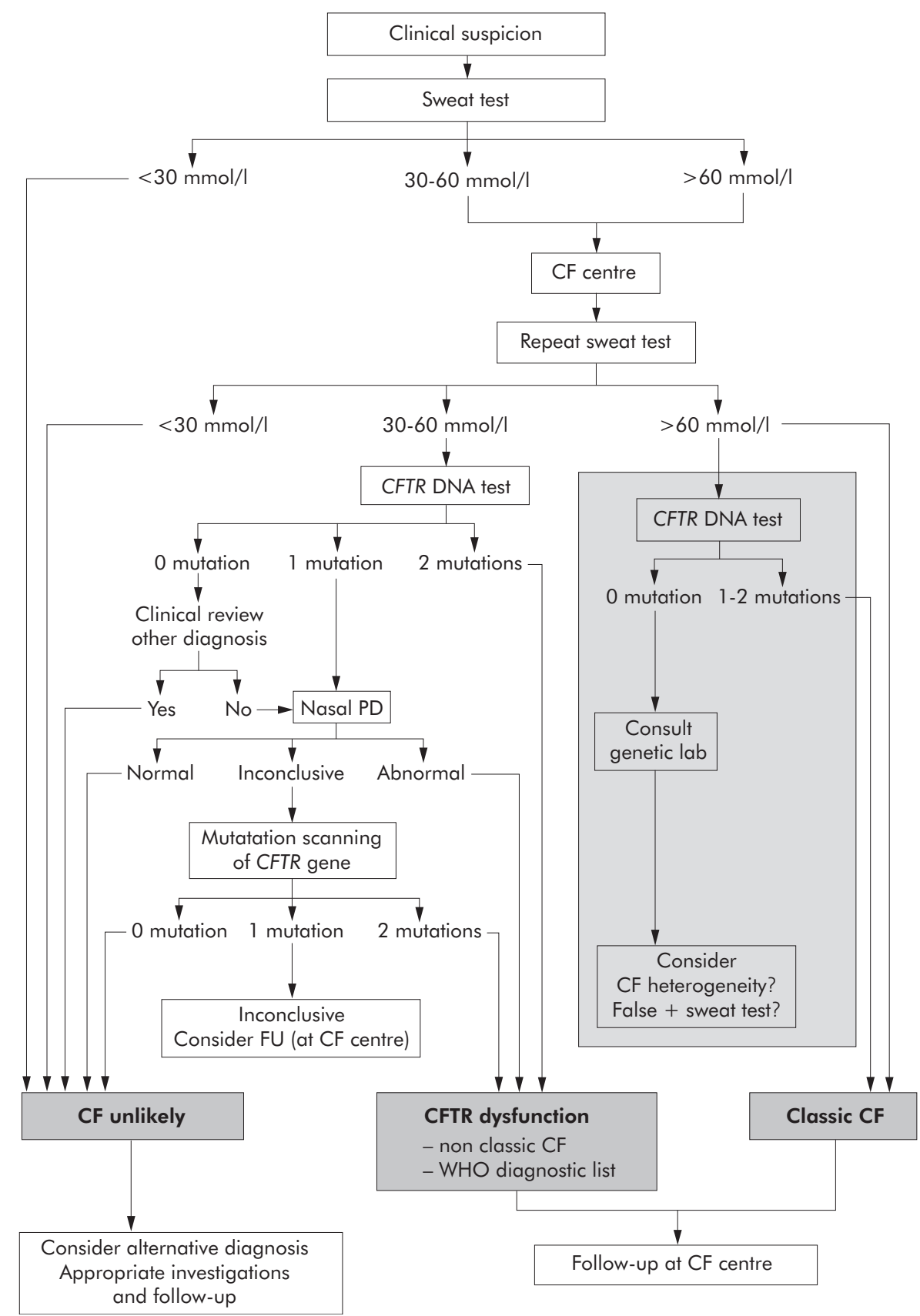

Figure 1 Algorithm for the diagnosis of CF starting with the sweat test. When entering the algorithm it is advised to continue the diagnostic work up if symptoms in a patient persist, as well as when symptoms have resolved but are highly suspicious for CF such as pancreatitis or Pseudomonas aeruginosa lung disease. Wherever the algorithm ends with "CF unlikely" it is advised to investigate for alternative diagnoses such as primary ciliary dyskinesia, humoral immunodeficiency, Shwachman syndrome. For patients with CFTR dysfunction, the physician needs to decide the most appropriate diagnostic label (non-classic CF or an item from the WHO diagnostic list shown in table 1 in patients with very limited symptoms). Patients with a borderline sweat test (30-60 mmol/l), only one CFTR mutation identified, and an inconclusive nasal potential difference (PD) cannot at present be classified correctly. They are at least CF carriers. In the presence of persistent symptoms they need structured follow up at an appropriate facility (for some patients this may be the CF centre) and symptomatic treatment. Genetic counselling is important in these patients and their families. CFTR DNA test: screening test to search for the most frequent mutations in the population from which the patient originates. Mutation scanning of CFTR gene: this test is only necessary in some patients in whom the diagnosis cannot be supported by other means. The tests in the grey area are optional because two clearly positive sweat tests are sufficient to support the diagnosis of CF in a compatible clinical setting. However, in most CF centres the CFTR DNA test will be performed to confirm the diagnosis, to allow for further cascade screening if necessary, and at times for research purposes. Consult genetic lab: in patients with an elevated sweat chloride level it would be unusual but not impossible not to find any mutation. In case of doubt about the diagnosis, a mutation scanning of the complete gene can be done. A falsely positive sweat test and the possibility of CF heterogeneity also need to be considered.

classified as "mild". Some patients with single organ involvement resulting from CFTR dysfunction may be more appropriately given an alternative "diagnostic label" as recommended in the World Health Organization (WHO) diagnostic list (table 1$).^{2}$ In these cases the importance of identifying a CFTR mutation may be greater for the family (for genetic counselling reasons) than for the index patient.

"Classic" and "typical" CF are considered synonyms; the same applies to "non-classic" and "atypical" CF. In the current document the terms "classic" and "non-classic" will 


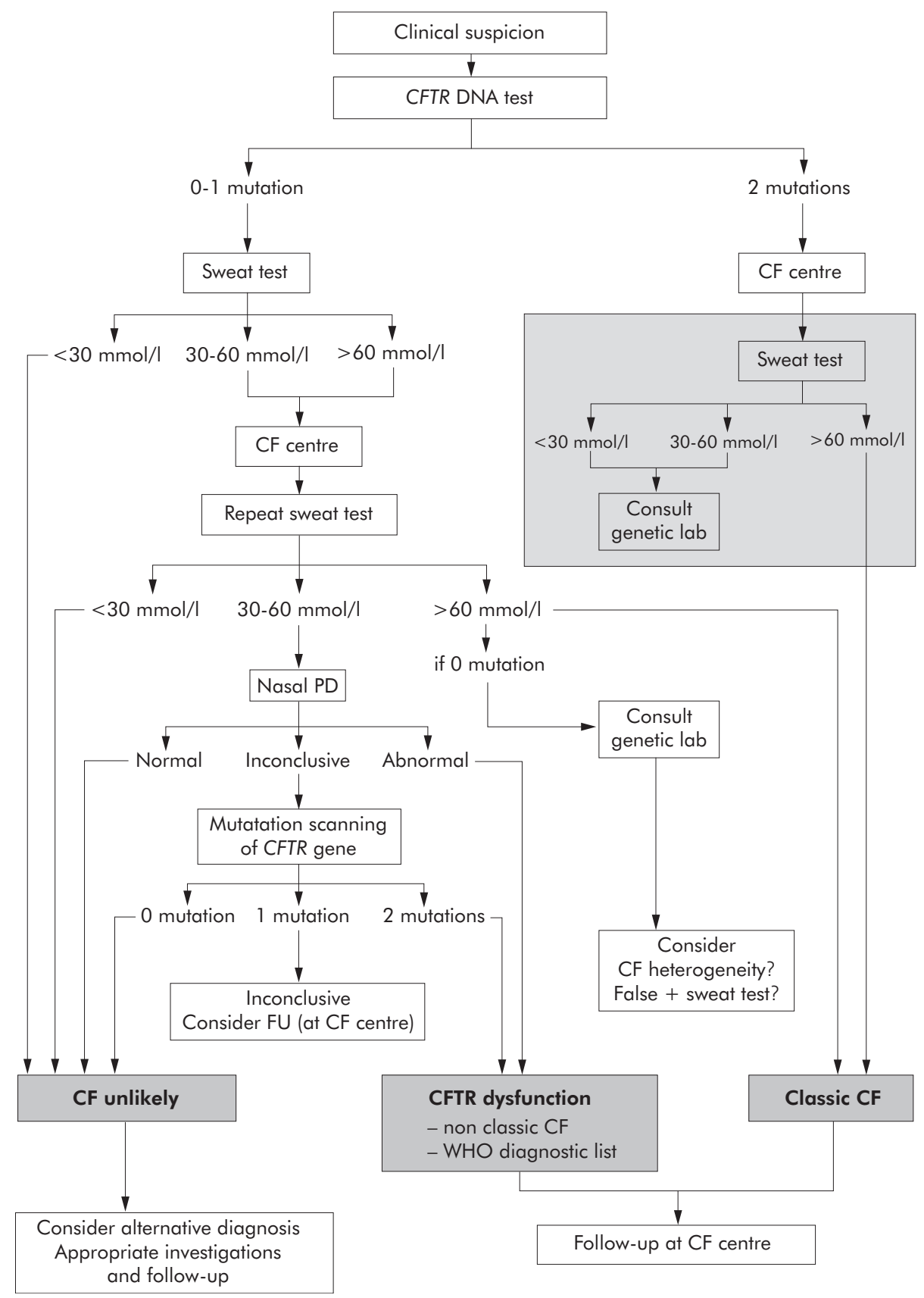

Figure 2 Algorithm for the diagnosis of CF starting with the CFTR DNA test. When entering the algorithm it is advised to continue the diagnostic work up if symptoms in a patient persist as well as when symptoms have resolved but are highly suspicious for CF such as pancreatitis or Pseudomonas aeruginosa lung disease. Wherever the algorithm ends with "CF unlikely" it is advised to investigate for alternative diagnoses such as primary ciliary dyskinesia, humoral immunodeficiency, Shwachman syndrome. For patients with CFTR dysfunction, the physician needs to decide the most appropriate diagnostic label (non-classic CF or an item from the WHO diagnostic list shown in table 1 in patients with very limited symptoms). Patients with a borderline sweat test, only one CFTR mutation identified, and an inconclusive nasal potential difference (PD) can not at present be classified correctly. They are at least CF carriers. In the presence of persistent symptoms they need structured follow up at an appropriate facility (for some patients this may be the CF centre) and symptomatic treatment. Genetic counselling is important in these patients and their families. CFTR DNA test: screening test to search for the most frequent mutations in the population from which the patient originates. Mutation scanning of CFTR gene: this test is only necessary in some patients in whom the diagnosis cannot be supported by other means. The tests in the grey area are optional because detection of two disease causing mutations is sufficient to support the diagnosis of CF in a compatible clinical setting. However, in many CF centres a sweat test will also be performed to further confirm this lifelong diagnosis. Consult genetic lab: in patients with two mutations detected it would be unusual to have a sweat chloride value below $60 \mathrm{mmol} / \mathrm{l}$ unless one or both mutations are classified as mild. One should not, however, forget the possibility of mislabelling a sample or errors in sweat test or DNA test; in patients with an elevated sweat chloride level it would be unusual but not impossible not to find any mutation. In case of doubt about the diagnosis, a mutation scanning of the complete gene can be done. A falsely positive sweat test and the possibility of CF heterogeneity also need to be considered.

be used. These definitions are helpful but one should not be rigid because the CF phenotype is a continuum of symptoms and cannot be hermetically defined in distinct disease categories. Apart from the CFTR mutations, modifier genes, lifestyle, treatment, environment and age all play a role in determining the phenotype. Our knowledge about the 


\begin{tabular}{l} 
Table 1 WHO diagnostic list for single organ \\
disease phenotypes associated with CFTR \\
mutations \\
\hline Isolated obstructive azoospermia* \\
Chronic pancreatitis* \\
Allergic bronchopulmonary aspergillosis* \\
Disseminated bronchiectasis* \\
Diffuse panbronchiolitis* \\
Sclerosing cholangitis* \\
Neonatal hypertrypsinogenaemia \\
\hline *At least one CFTR mutation identified. \\
It is likely that this classification will need further revision in \\
the future as our knowledge and understanding of these \\
conditions increase.
\end{tabular}

interplay of these factors is at present very incomplete. ${ }^{36}$ Researchers and specialists in the field are aware of these facts, but these factors are not quantifiable in daily practice. It is clinically useful to distinguish between classic (typical) $\mathrm{CF}$ and non-classic (atypical) CF. The best criterion to make this crude distinction is the sweat test which has been used for decades and remains the gold standard for clinical diagnosis. ${ }^{15}$ No clinician will doubt the diagnosis of CF in a patient with compatible symptoms if a correctly performed sweat test reveals a chloride value above $60 \mathrm{mmol} / \mathrm{l}^{7-9}$ The same pragmatic distinction between classic and non-classic CF based on sweat test results has been made before by Boyle $^{4}$ and, until other data become available, the sweat test result provides the most practical and most reliable distinction. Since the discovery of the CFTR gene, CFTR mutation analysis and diagnostic tests that measure chloride channel activity have become available. ${ }^{10}$ Subjects with some CF disease characteristics and sweat chloride values below the $60 \mathrm{mmol} / \mathrm{l}$ cut off but carrying two CFTR mutations have been identified. ${ }^{11}{ }^{12}$ For several rare mutations it is unclear if they really are mutations or what impact they have on the phenotype. ${ }^{13}$ To date, no test has proved to be as practical or reliable as the sweat test for clinical diagnostic purposes. ${ }^{7}{ }^{9}$

As is clear from the definitions of classic and non-classic $\mathrm{CF}$, the terms "mild versus severe" and "single organ disease versus multi organ disease" are rather subjective and should only be used as descriptive terms; they are not intrinsically linked to classic and non-classic CF. Since treatment has improved over time, an 18 year old homozygous F508del patient with a sweat chloride level of $105 \mathrm{mmol} / \mathrm{l}$ may have only very mild disease-that is, normal height and weight, normal lung function, and minimal changes on the chest CT scan. Conversely, a patient with D1152H plus F508del with a sweat chloride level of $45 \mathrm{mmol} / \mathrm{l}$ may have escaped early diagnosis and may have had several episodes of lower respiratory tract disease leading to widespread bronchiectasis by the age of 18 years. Usually, however, patients with nonclassic disease are more likely to have mutations regarded as less severe. ${ }^{12}$ Single organ disease means that disease or dysfunction is detected in only one organ, but this may change with age and, with the use of more sensitive tests, disease manifestations may be detected in several organs. Clinically, single organ disease is more common in patients with non-classic CF but can occur in patients with what is otherwise classic disease-for example, a 10 year old patient with pancreatic sufficiency who has recurrent respiratory tract infections.

The diagnostic confirmation of CF can be made by DNA analysis of CFTR mutations or by a sweat test. A "first line" CFTR DNA test should screen for the CF causing CFTR mutations that are most prevalent in the population from which the patient originates-that is, CFTR mutations with a frequency higher than $0.5 \%$. In most populations a mutation detection rate of $80-95 \%$ is thus obtained..$^{13}$ Using the sweat test an even higher detection rate is obtained-for example, 98\% of American CF patients have an elevated sweat chloride level. ${ }^{1}$ Therefore, in settings where the reliable Gibson and Cooke sweat test is available, the approach starting with the sweat test (fig l) is the preferred route. In patients with atypical disease manifestations the reliability of the sweat test is much lower and additional diagnostic tests will be necessary to substantiate the diagnosis; the algorithm starting with DNA analysis (fig 2) could then be more appropriate.

\section{INDICATIONS TO ENTER THE CF DIAGNOSTIC ALGORITHM}

Three main situations set off the CF diagnostic cascade: (1) clinical manifestations; (2) neonatal screening; and (3) family history.

\section{Clinical manifestations}

Many clinical problems are compatible with a diagnosis of CF because this multiorgan disease is very heterogeneous and has, at times, an atypical clinical presentation. ${ }^{14}$ In patients with the classical clinical picture the diagnostic tests are mainly needed to confirm the diagnosis. In patients with mild or atypical symptoms or in patients with single organ disease, the diagnostic tests are necessary to support or exclude the diagnosis of $\mathrm{CF}$ or to point to an alternative diagnosis.

Some phenotypic features are indeed highly suggestive for CF (table 2, left column) and should always lead to further investigation. If initial investigations such as a sweat test and a standard CFTR mutation screening do not support the diagnosis of $\mathrm{CF}$, and a definite alternative diagnosis is not found, progression of the work up is justified. Whenever symptoms may be caused by CF, it is very important that a timely diagnosis is made.

Other features may be less specific to CF since they are also commonly associated with other medical conditions such as humoral immunodeficiency or primary ciliary dyskinesia (table 2, right column). Other specific tests should be used to better define the clinical picture including chest radiography, pulmonary function testing, sputum culture, determination of fecal chymotrypsin or elastase, and a spermogram in adult men.

Only rarely do patients have CF phenotypic features in the face of a sweat chloride below $30 \mathrm{mmol} / \mathrm{l}$; one such example is the mutation $3849+10 \mathrm{kbC}>\mathrm{T} .{ }^{11}$ The complex issue of CF heterogeneity-that is, CF-like disease not caused by CFTR mutations - still needs to be resolved. In the cohort reported to date, the majority of these patients had a sweat chloride value above $40 \mathrm{mmol} / \mathrm{l} .{ }^{14}$

$\mathrm{CF}$ is nearly always a clinical diagnosis. However, in a neonatal screening programme or in a sibling of a known patient, the diagnosis of CF may come before the child has shown any symptoms. It is unlikely that a similar situation would arise in teenagers or adults, except in cases detected through cascade screening.

\section{Neonatal screening}

CF neonatal screening is based on the immunoreactive trypsinogen (IRT) assay, which is relatively inexpensive and adaptable to large numbers. ${ }^{15}$ Increased IRT concentrations at birth are characteristic of newborns affected by CF, but can also be found in healthy infants. IRT values tend to remain raised for several months in newborns with $\mathrm{CF}$, whereas in false positives they usually return to normal within the first weeks of life. To improve the specificity of neonatal screening, a second blood sample is obtained in neonates with raised levels of IRT at birth, and only infants with persistently 


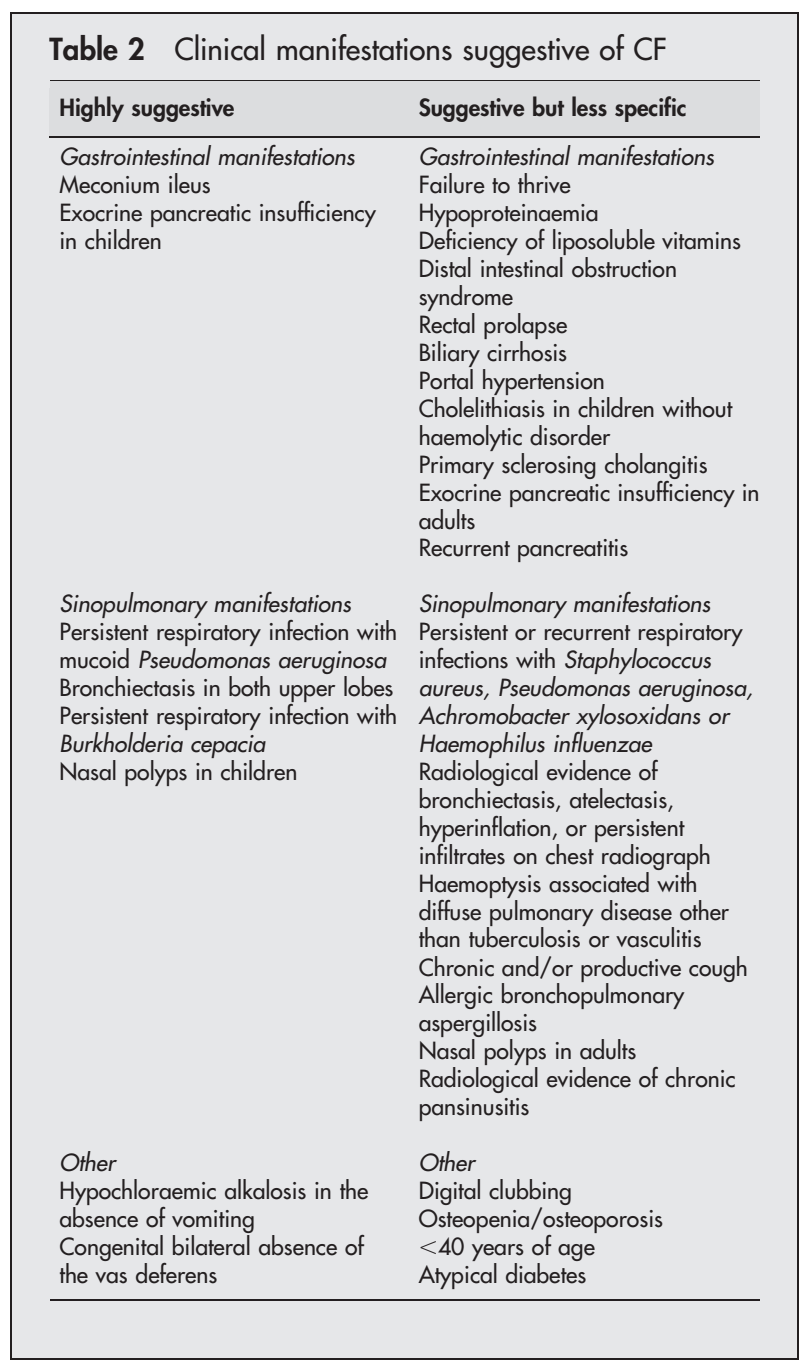

raised IRT values progress to a sweat test. Nowadays, in most neonatal screening protocols, IRT retesting in infants with an initially raised value has been replaced by analysis of a panel of CF causing mutations in the neonatal blood sample. ${ }^{16}$ Homozygotes and compound heterozygotes are considered affected (a confirmatory sweat test is anyway desirable), while babies carrying one mutation progress to a sweat test in order to distinguish affected individuals from carriers. Screened babies may have some early manifestations of the disease or be completely asymptomatic.

\section{Family history}

It is strongly advised that siblings of affected children are investigated by a sweat test. Because of the remarkable clinical heterogeneity, even within families, lack of symptoms is insufficient to exclude the diagnosis of CF. If the affected sibling has a clearly positive sweat test, the algorithm in fig 1 presents the optimal diagnostic approach. Beginning the diagnostic procedure with DNA testing is less desirable because detection of the carrier status in minors confers no benefit for the individual and possibly leads to stigmatisation. ${ }^{17}$ Knowledge of carrier status is indeed only of significance for reproductive questions in the future. If the affected sibling has a sweat chloride that is borderline or negative, the algorithm in fig 2 should be used. When the full CFTR genotype of the affected child is unavailable, segregation analysis of markers within or linked to the CFTR gene may be an option in that family.
Other relatives of CF patients should be offered appropriate genetic counselling. Likewise, when both partners carry a CFTR mutation, they should be referred for prenatal counselling.

\section{SWEAT TEST}

The development of the quantitative pilocarpine iontophoresis by Gibson and Cooke dates from 1959 and is still the test of choice. ${ }^{18}$ Normal sweat contains less than $60 \mathrm{mmol} / \mathrm{l}$ chloride and sodium. A sweat chloride level above $60 \mathrm{mmol} / \mathrm{l}$ in the absence of CF is rare, although it has been reported in a number of unusual clinical conditions that can usually be readily distinguished from CF. ${ }^{9}$ In infants the upper limit of normal may be lower $(40 \mathrm{mmol} / \mathrm{l}$ which corresponds to the mean +3 SD of the heterozygote carrier group).${ }^{19}$ Testing can be carried out after the first 2 weeks of life in infants weighing more than $3 \mathrm{~kg}$ who are normally hydrated and without significant illness. Testing should be delayed in infants who are acutely ill or dehydrated, who have eczema or oedema, or who are receiving supplemental oxygen. Raised sweat electrolyte concentrations can be found in infants who are underweight or dehydrated. Systemic steroids and oedema can lower sweat electrolyte concentrations. Sweat electrolytes are not affected by intake of flucloxacillin, diuretics or administration of intravenous fluids. For all these and other details about the sweat test we refer to recent overviews..$^{7-9}$

Chloride concentration measurement is the analysis of choice because the chloride ion concentration shows the greatest discrimination between CF and normal subjects. Moreover, it has become clear that chloride is the ion most directly related to CFTR dysfunction. Concurrent measurement of sodium acts as a quality control. In CF individuals the sweat chloride is usually higher than the sweat sodium, but the converse is true in normal persons. ${ }^{7}$

Pilocarpine iontophoresis is the preferred method of sweat stimulation. $^{7-9} 1820$ Sweat should be collected for 30 minutes onto preweighed gauze or filter paper low in sodium chloride. A minimum sweat rate of $1 \mathrm{~g} / \mathrm{m}^{2}$ body surface area/min is required; thus a sweat volume of $50-100 \mathrm{ml}$ is adequate.

Since the discovery of the CFTR gene, it has become clear that a proportion of subjects carrying two CFTR gene mutations have a sweat chloride level below $60 \mathrm{mmol} / \mathrm{l} .{ }^{11}{ }^{12}$ Most of the studies exploring these patients with equivocal sweat tests have focused on the chloride range $40-60 \mathrm{mmol} /$ $1 .{ }^{21}$ In the UK guidelines on sweat testing, ${ }^{7} 40 \mathrm{mmol} / \mathrm{l}$ is considered as the lower limit for equivocal sweat tests because this value represents the mean $+2 \mathrm{SD}$ in carriers. The level of evidence for the data supporting this statement was not graded as high (only evidence $\mathrm{B}$ level $2 \mathrm{~b}$ and 3 ). Indeed, the majority of studies referred to in the UK document date from the time before genotype analysis and, as stated in the document: "the normals could include some persons with CF or CF related disorders".

The evidence that a proportion of CF patients with chloride concentrations of $30-60 \mathrm{mmol} / \mathrm{l}$ will be found to have two CFTR mutations is recent and has evolved following CFTR mutation testing. ${ }^{22}$ Sweat chloride concentrations of 30$60 \mathrm{mmol} / \mathrm{l}$ are seen in about $4 \%$ of sweat tests; $23 \%$ of these patients will subsequently be found to have two CFTR mutations. CF affected patients occur with similar frequency in the $30-40 \mathrm{mmol} / \mathrm{l}$ range as in the $40-60 \mathrm{mmol} / \mathrm{l} \mathrm{range} \mathrm{e}^{22}$ so, in the algorithms presented in figs 1 and 2, a chloride cut off level of $30 \mathrm{mmol} / \mathrm{l}$ is chosen.

\section{CFTR GENE MUTATIONS}

The CFTR gene, located on the long arm of chromosome 7, encodes for the CFTR protein that functions as a cAMP 
mediated chloride channel and regulates ion and water balance across epithelia. ${ }^{23}$

Over 1400 CFTR mutations have been identified, ${ }^{24}$ of which more than 1000 are known to cause CF while the remainder are involved in the milder CFTR related diseases or do not cause disease at all. In the Rosenstein consensus, ${ }^{5}$ a CF causing mutations is defined as an alteration in the CFTR gene that fulfils one of the following criteria: (1) causes a change in the amino acid sequence that severely affects CFTR synthesis or function; (2) introduces a premature termination signal; (3) alters the "invariant" nucleotides of intron splice sites; (4) causes a novel amino acid sequence that does not occur in the normal CFTR genes from at least 100 carriers of CF mutations from the patient's ethnic group. For several mutations-that is, most class I mutations (nonsense mutations, splice site mutations, out frame deletions/ insertions)-the CF causing nature is obvious. For the remaining mutations, only functional studies unequivocally determine if they are CF causing mutations. So far this has only been done for the most common CFTR mutations. A rare missense mutation found after complete screening of the CFTR gene can be either a polymorphism or a CF causing mutation.

Most mutations are point mutations-that is, only one nucleotide is mutated. A CF patient can either be homozygous (carrying an identical CFTR mutation on both CFTR alleles) or compound heterozygous (have two different CFTR mutations). The distribution of CFTR mutations differs between different ethnic populations. ${ }^{13}$ The most common mutation, F508del, reaches frequencies of $70 \%$ or more in northern European populations, with lower frequencies in southern European populations. Other common mutations exist in most populations, each reaching population frequencies of about 1-2\%. Examples include the G542X, G551D, R553X, W1282X and N1303K mutations. Finally, for a given population, ethnic specific mutations that reach frequencies of about $1 \%$ up to $7 \%$ might exist. For most populations, all these common mutations cover about $80-95 \%$ of all mutant CFTR genes.

Several commercial assays are available for CFTR mutation screening, such as the OLA Cystic Fibrosis Assay (Abbott Laboratories), the INNO-LiPA CFTR Assay (Innogenetics), and the Elucigene CF Assay (Tepnel Diagnostics). Most tests only screen for about 30 mutations, the majority of which are associated with classic CF. ${ }^{25}$

A mutation detection rate of $90 \%$ in a specific population signifies that a mutation will be identified on both CFTR genes in $81 \%$ of the typical CF patients; a mutation will be found on only one CFTR gene in $18 \%$; and no mutation will be found on either CFTR gene in $1 \%$. In the case of a borderline sweat test, extensive mutation screening of both CFTR genes may be required with assays such as DGGE (denaturing gradient gel electrophoresis), dHPLC (denaturing high pressure liquid chromatography), SSCP (single strand conformation polymorphism assay), and sequencing. ${ }^{26}{ }^{27}$ Only sequencing will approach $100 \%$ sensitivity. The other techniques are indirect mutation scanning assays with sensitivities varying from close to $100 \%$ to as low as $90 \%$. Even if a mutation is found, its involvement in disease may not be clear. For many CFTR mutations the functional consequences are unknown; they may even be polymorphisms. ${ }^{24}$ These assays only screen for mutations in the coding region and exon/intron junctions of the CFTR gene. Mutations located deep in the promoter or introns, as well as deletions and insertions of one or more exons, may remain undetected. In northern Europe, large deletions in the CFTR gene are found in $15 \%$ of CF patients in whom no mutations were found after extensive scanning of the complete CFTR coding region-that is, in about $0.3 \%$ of all CF patients. ${ }^{28}$
Such large CFTR deletions can be detected by means of a MLPA (Multiplex Ligation-dependent Probe Amplification) assay (MRC, Holland) and are more likely to be found in patients with classic CF. Missed deep intronic mutations and promoter mutations are more likely to be mild mutations and of relevance in patients with non-classic CF.

At the end of intron 8 of the CFTR gene, a stretch of 5, 7, or 9 thymidine residues is found at the Tn locus, designated T5, T7 and T9 allele. A lower number of thymidines results in less efficient splicing of CFTR transcripts, and therefore a lower amount of functional CFTR protein. The T5 allele has been classified as a mild disease mutation with partial penetrance. In the general white ethnic population the T5 polymorphism is found on about $5 \%$ of the CFTR genes, but on about $21 \%$ of the CFTR genes derived from patients with congenital bilateral absence of the vas deferens (CBAVD), ${ }^{29}$ and it may even confer non-classic CF. ${ }^{30}{ }^{31}$ In most cases the partial penetrance is explained by the polymorphic TGm locus (11, 12 or 13 TG repeats) in front of the T5 allele; a higher number of TG repeats also results in less efficient splicing of CFTR transcripts. ${ }^{30}$ In the general white ethnic population, of all T5 CFTR genes, the less harmful TG11-T5 CFTR gene is found at a frequency of about $80 \%$. In healthy individuals, who are compound heterozygous for a severe mutation and the T5 allele (for example, fathers of CF patients), T5 is associated with the milder TGll allele. ${ }^{30}$ In patients with CBAVD and non-classic CF, the milder TG11-T5 allele is infrequent while the TG12-T5 allele is most frequently found. ${ }^{30}{ }^{32}$ The TG13-T5 is rarer but also more frequently found in patients with CBAVD and non-classic CF. ${ }^{30}$ Most commercial tests screen for the T5 allele. Since most T5 alleles carry a non-harmful TG11 allele, conclusions of a possible involvement of T5 in disease can only be obtained after analysis of the TGm locus which can currently only be accurately determined by sequencing.

In patients with classic CF and only one CF causing CFTR mutation, further DNA testing is not mandatory. Prenatal diagnosis and carrier screening of relatives can be performed by segregation analysis of polymorphisms within or linked to the CFTR gene.

In patients with typical CF symptoms but no disease causing mutations detected, genetic heterogeneity has to be considered. Indeed, although rare, the CF phenotype may be caused by a genetic factor other than CFTR. ${ }^{14}{ }^{33}$ If another gene is involved in the disease, prenatal diagnosis and carrier screening by segregation analysis of polymorphisms within or linked to the CFTR gene will result in false conclusions. To prevent this type of error, extensive mutation scanning of the complete CFTR genes is recommended in some families to prove or disprove the involvement of CFTR in the particular subject.

\section{CFTR BIOASSAYS}

The "disease" of CF results from defective cAMP induced chloride secretion through mutated CFTR protein, combined with compensatory excessive sodium influx into epithelial cells. ${ }^{23}{ }^{34}$ CFTR bioassays measure the epithelial ion fluxes or their resulting voltage potential at the mucosal surface. ${ }^{10}$ These assays thus provide a direct view of the physiology at the cellular and the ion channel level. They help to resolve diagnostic dilemmas in atypical patients by ruling in or out a CFTR related dysfunction. The tests can be done on respiratory or intestinal epithelium. They can be performed in vivo (nasal or bronchial potential measurement) or ex vivo on intestinal mucosal samples or airway cell cultures. In many centres nasal potential difference (PD) measurements and/or intestinal current measurements are not available. In these settings the diagnostic algorithm can skip this step. 

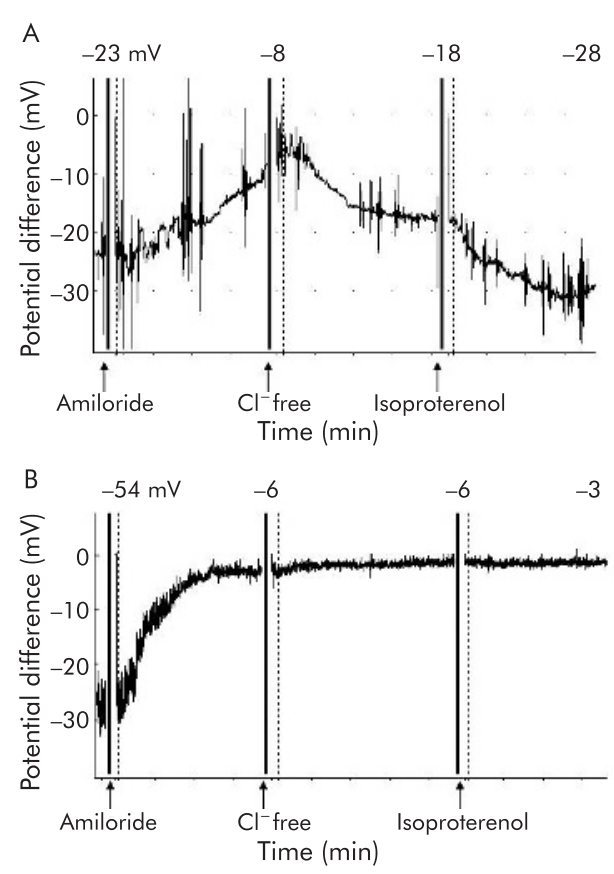

Figure 3 Nasal potential difference measurement in (A) a healthy person and (B) a person with classic CF. In a healthy person the basal PD is negative $(-20$ to $-30 \mathrm{mV}$ ), rises moderately (towards zero) after application of amiloride, and decreases after application of chloride free solution and isoproterenol. In a patient with typical CF the baseline PD is more negative and the rise after application of amiloride is greater. There is no change in PD after application of chloride free solution and isoproterenol.

\section{Transepithelial nasal potential difference (nasal PD)}

The use of nasal PD as a diagnostic test for CF is already accepted in the diagnostic consensus of Rosenstein et al. ${ }^{5}$ The test has been introduced in clinical practice in many large CF centres that have compiled their own reference values. Several papers report on the usefulness of nasal PD measurements for diagnosing $\mathrm{CF}^{35-38}$

Nasal PD is determined by standard criteria as described by Knowles et al. ${ }^{10}$ The PD is measured between a fluid filled exploring bridge on the nasal mucosa and a reference bridge on the skin of the forearm. The reference bridge may be applied to the skin by a thin needle inserted subcutaneously or placed directly on the skin after performing a small abrasion. Practical details of this test have been published elsewhere. ${ }^{10}{ }^{39}$ With skilled operators and careful attention to technical details, the nasal PD measurement can be used as an outcome measure in therapeutic trials. ${ }^{40}$

The basal PD gives an indication of sodium transport via the amiloride sensitive epithelial sodium channel. After consistent baseline PD measurements have been obtained, the effect of amiloride superfusion through a second tube overriding the exploring catheter is evaluated. To study nasal chloride permeability and cAMP activation of chloride permeability, a large chloride chemical gradient is generated across the apical membrane by superfusion of the nasal mucosa for 3 minutes with a chloride free solution containing $10^{-4} \mathrm{M}$ amiloride in Ringer's solution with gluconate substituted for chloride at a rate of $5 \mathrm{ml} / \mathrm{min}$. The same solution to which isoproterenol $10^{-5} \mathrm{M}$ has been added is perfused for a further 3 minutes. The change in voltage response over the final 6 minutes serves as an index of cAMP activation of epithelial chloride permeability.

The nasal PD of a patient with classic CF is remarkably different from controls (fig 3). The basal PD is much higher (more negative), the amiloride response is exaggerated, and there is very little or no response to chloride free and isoproterenol solutions. In non-classic CF the nasal PD may be borderline and there is not yet a total consensus as to what exactly constitutes an abnormal result, but a formula which takes into account both sodium and chloride transport has been proposed. ${ }^{41}$

\section{Intestinal current measurement (ICM)}

The abnormalities in epithelial ion transport characteristic of $\mathrm{CF}$ are also expressed throughout the intestinal tract. The intestinal epithelium does not undergo infection induced degenerative changes and is therefore suitable for use as a functional test for the disease. ${ }^{42}$ As many intestinal ion transport processes are electrogenic, measuring the electrical current that they generate (intestinal current measurement, ICM) can be used to monitor their activity. In CF, intestinal chloride secretion is impaired while absorptive processes remain unchanged and may even be enhanced. Measurements can be performed on $\operatorname{rectal}^{43-45}$ or jejunal ${ }^{46}$ mucosa. There is a clear difference between ICM measurement in classic CF and in normal individuals (fig 4). Data are only now emerging about the use of ICM as a clinical
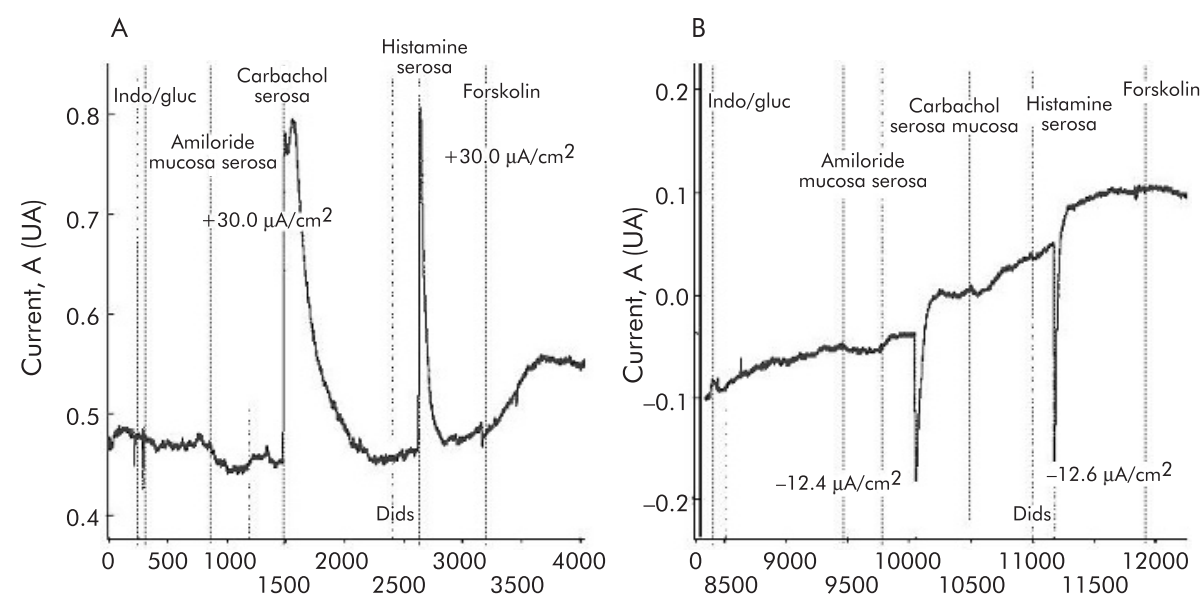

Figure 4 Intestinal current measurement in (A) a healthy person and (B) in a patient with classic CF. The responses to carbachol and histamine consist of two components: a lumen positive current that is most likely caused by the apical potassium efflux and a lumen negative current caused by apical chloride secretion. In ICM measurements of non-CF individuals the apical potassium efflux component in reaction to carbachol and histamine is masked by the much larger chloride efflux. In CF the response is reversed due to the apical potassium efflux in the absence of a chloride efflux. 
diagnostic tool. ${ }^{44}{ }^{47}$ At present the technique has remained mainly in the research setting, so it is not yet included in the diagnostic algorithms.

\section{HOW TO USE THE ALGORITHMS}

The WHO Working Group of $2001^{2}$ stressed that the diagnosis of CF is ultimately clinical, but laboratory investigations are important tools which support the clinical assessment or help in difficult cases. The algorithms presented here are offered in that same spirit. They are a pathway to diagnosis, without claiming that they provide absolutely foolproof tools: the clinician may still have to make a final decision based on experience and the weight of evidence.

The phenotypic expression of CF is variable. Many symptoms can be the first sign of this multisystem disease. The symptoms compatible with a clinical phenotype of CF are listed in table 2 and can each be the reason for starting the diagnostic algorithm. A positive neonatal screening test and a family history of CF are alternative starting points. The diagnostic algorithms lead to three possible outcomes: (1) CF unlikely; (2) CFTR dysfunction; and (3) classic CF. Within CFTR dysfunction the physician needs to decide whether the patient is best described as non-classic CF or with an item from the WHO diagnostic list for patients with very limited symptoms. The more one moves to the right side of the algorithm, the more classic CF becomes likely; the more one moves to the left side of the algorithm, the less likely CF becomes.

\section{Algorithm starting with the sweat test (fig 1)}

As stated above, when the reliable sweat test according to Gibson and Cooke is available, the algorithm presented in fig 1 is preferred. The sweat test is cheap and, in nearly all populations, will result in a greater diagnostic yield than a standard CFTR DNA screening test.

All patients with a sweat chloride level above $60 \mathrm{mmol} / \mathrm{l}$ and a clinical phenotype compatible with CF have a diagnosis of classic CF. The grey area in the algorithm indicates tests that are suggested but not obligatory. As an extra precaution, because $\mathrm{CF}$ is a lifelong diagnosis and because errors may occur, it is suggested that, in addition to sweat testing, mutation analysis is done to further confirm the diagnosis of CF. Only if no mutations are found should the possibility of CF heterogeneity be considered. ${ }^{14}$ A sweat chloride level above $60 \mathrm{mmol} / \mathrm{l}$ has also been reported in a few very rare conditions that are usually easily differentiated from CF. ${ }^{7}$

In patients with a sweat chloride level below $30 \mathrm{mmol} / \mathrm{l}$, the diagnosis of CF becomes very unlikely.

Patients with an indeterminate sweat chloride level (30$60 \mathrm{mmol} / \mathrm{l}$ ) require further investigation. When no mutations are found in this group, it is wiser to look for alternative diagnoses and to treat the patient appropriately. In case of respiratory symptoms, the search for an alternative diagnosis will include, among others allergy tests, evaluation of humoral immunodeficiency, gastro-oesophageal reflux, and ciliary function. In patients in whom only one CFTR mutation can be detected, in patients with ongoing symptoms, or those in whom no alternative diagnosis is retained, one may proceed with CFTR sequencing. The cost of gene sequencing is high so centres with access to nasal PD measurements can further narrow down the group in whom genomic sequencing is necessary by limiting sequencing to patients with an inconclusive nasal PD tracing.

Patients with an indeterminate sweat chloride, only one mutation identified, and an inconclusive nasal PD measurement pose the real diagnostic challenge; at present they cannot be classified because CFTR dysfunction has not been clearly demonstrated. They are at least CF carriers. In the presence of persistent symptoms they need structured follow up at an appropriate facility (for some patients this may be the CF centre) and symptomatic treatment. Genetic counselling is important in these patients and their families. Long term information about this type of patient needs to be collected and improved understanding is likely to follow; the combination of one mutation plus several unfavourable modifier genes is one possibility.

\section{Algorithm starting with the CFTR DNA test (fig 2)}

The diagnostic algorithm in fig 2 is used mainly in neonatal screening programmes and in cascade screening. Identifying a disease causing mutation in both CFTR genes is necessary to conclude the diagnosis of CF. The need to confirm a diagnosis based on identification of two CFTR mutations by a sweat test can be debated (optional "grey" area in the algorithm). Since $\mathrm{CF}$ is a diagnosis for a lifetime, extreme care should be taken that a diagnosis based on only one diagnostic test is totally reliable. Mistakes with DNA mutation analysis may occur. ${ }^{25}$ When none or one mutation is detected, the sweat test is indicated. Similar to the algorithm in fig 1, it is again in the group with a sweat chloride level of $30-60 \mathrm{mmol} / \mathrm{l}$ that further tests are appropriate (nasal PD if available and mutation scanning if nasal PD is not available or inconclusive).

Evidence of a CFTR dysfunction by nasal PD measurement or even by a sweat test does not of itself mean that the appropriate diagnosis is CF. The diagnosis is made on the clinical picture first. The tests only support the clinical diagnosis. If CFTR dysfunction has been documented but the clinical picture is limited-for example, male infertility as in some cases of CBAVD-it is better to use an alternative diagnosis as suggested in the WHO recommendations (table 1). ${ }^{2}$ The best diagnosis in this example will be CBAVD provided there is no clinical evidence of disease in another organ system. All that the genetic and physiological tests have added is to provide an aetiological cause for the CBAVD - that is, CFTR dysfunction in that organ; they have not changed the clinical diagnosis to CF. Follow up of these patients is necessary for two reasons: (1) the clinical features in the individual patient may change over time-for example, someone with isolated CBAVD may develop pulmonary infections and the appropriate diagnosis may change to non-classic CF; and (2) clinicians need to expand and share their understanding about what CFTR dysfunction means for the lifetime of a patient.

\section{CONCLUSIONS}

In the majority of cases the diagnosis of $\mathrm{CF}$ is clearcut; the clinical picture is obvious and the clinical diagnosis is supported by the sweat test result as well as by mutation analysis. Non-classic CF in children and young adults provides a greater challenge. Fortunately, in many cases, further CFTR mutation analysis and bioassays of chloride secretion will support or exclude the diagnosis. The need for a structured diagnostic process is obvious in such a complex disease. The definitions and algorithms presented in this paper are the product of lengthy discussions between experts in the field of CF diagnosis.

More data are needed about CF heterogeneity. The creation of a diagnostic network with international collaboration will help to collect reliable clinical and laboratory data in patients with CF-like symptoms without a clearcut diagnosis of CF and will hopefully identify new clinical disease patterns and aetiologies.

Even when a definitive diagnosis cannot be made in some patients, intensive supportive treatment and follow up must be started when symptoms persist. The situation is more difficult in neonates who do not yet have symptoms and in whom nasal PD may be difficult to measure. An increased 
IRT, one CFTR mutation, and a borderline sweat test result in a newborn may well be suggestive of non-classic CF. Such a child should be followed up closely and possibly put on preventive treatment. These subjects probably have a much better prognosis. Long term follow up of such a cohort is necessary to support an evidence based correct diagnosis and individualised treatment for similar patients in the future.

\section{Authors' affiliations}

K De Boeck, Department of Pediatrics, University of Leuven, Belgium M Wilschanski, Department of Pediatric Gastroenterology, Hadassah Medical Organization, Jerusalem, Israel

C Castellani, CF Centre, Ospedale Civile Maggiore, Verona, Italy C Taylor, Academic Unit of Child Health, University of Sheffield, UK H Cuppens, Department of Human Genetics, University of Leuven, Belgium

J Dodge, Department of Child Health, University of Wales, Swansea, UK M Sinaasappel, Department of Pediatrics, Erasmus MC-Sophia Children's Hospital, University Medical Centre, Rotterdam, The Netherlands

Funding: none

Competing interests: none declared.

Diagnostic Working Group: M Ballmann, Pediatric Department, CF Centre, Medical School, Hannover, Germany; I Bronsveld, Erasmus MC, Rotterdam, The Netherlands; $\mathrm{H}$ de Jonge, Erasmus MC, Rotterdam, The Netherlands; Y de Rijke, Erasmus MC, Rotterdam, The Netherlands; L Hielte, Stockholm CF Centre, Karolinska University Hospital Huddinge, Sweden; T Leal, Université Catholique de Louvain, Brussels, Belgium; M J Schwarz, St Mary's Hospital, Manchester, UK; I Sermet, Hôpital NeckerEnfants Malades, Paris, France; K Southern, Royal Liverpool Children's Hospital, Liverpool, UK; B Strandvik, Göteborg University, Göteborg, Sweden; B Tümmler, Medizinische Hochschule Hannover, Hannover, Germany

\section{REFERENCES}

1 Orenstein BM, Winnie GB, Altman H. Cystic fibrosis: a 2002 update. J Pediatr 2002; 140:156-64.

2 World Health Organization. Classification of cystic fibrosis and related disorders, Report of a Joint Working Group of WHO/ICF(M)A/ECFS/ECFTN, 2001 (reprinted in J Cyst Fibros 2002;1:5-8).

3 Goss CH, Rosenfeld M. Update on cystic fibrosis epidemiology. Curr Opin Pulm Med 2004; 10:510-4.

4 Boyle MP. Nonclassic cystic fibrosis and CFTR-related diseases. Curr Opin Pulm Med 2003;9:498-503.

5 Rosenstein BJ, Cutting GR, for the Cystic Fibrosis Foundation Consensus Panel. The diagnosis of cystic fibrosis: a consensus statement. J Pediatr 1998; 132:589-95.

6 Davies J, Alton E, Greisenbach U. Cystic fibrosis modifier genes. J R Soc Med 2005;98(Suppl 45):47-54

7 Association of Clinical Biochemistry. Guidelines for the performance of the sweat test for the investigation of cystic fibrosis in the UK, Report from the Multidisciplinary Working Group, 2002. Available at http:// www.acb.org.uk

8 National Committee for Clinical Laboratory Standards (NCCLS). Sweat testing: sample collection and quantitative analysis, Approved guideline C34A2. Wayne, PA: NCCLS, 2000.

9 Rosenstein BJ. Diagnostic methods. In: Hodson M, Geddes D, eds. Cystic fibrosis. 2nd ed. Arnold Publishers, 2000:177-88.

10 Knowles MR, Paradiso AM, Boucher RC. In vivo nasal potential techniques and protocols for the assessment of the efficiency of gene transfer in cystic fibrosis. Hum Gene Ther 1995:6:445-55.

11 Highsmith WE, Burch LH, Zhou Z, et al. A novel mutation in the cystic fibrosis gene in patients with pulmonary disease but normal sweat chloride concentrations. N Engl J Med 1994;331:974-80.

12 Cystic Fibrosis Genotype-Phenotype Consortium. Correlation between genotype and phenotype in patients with cystic fibrosis. N Engl J Med 1993;329:1308-13.

13 World Health Organization. The molecular genetic epidemiology of cystic fibrosis, 2004. Available at http://www.who.int/genomics/publications/en.

14 Groman JD, Meyer ME, Wilmott RW, et al. Variant cystic fibrosis phenotypes in the absence of CFTR mutations. N Engl J Med 2002;347:401-7.

15 Crossley JR, Smith PA, Edgar BW, et al. Neonatal screening for cystic fibrosis, using immunoreactive trypsin assay dried blood spots. Clin Chim Acta 1981;113:111-21.

16 Ranieri E, Lewis BD, Gerace RL, et al. Neonatal screening for cystic fibrosis: using immunoreactive trypsinogen and direct gene analysis: four year's experience. BMJ 1994;308:1469-72.
17 Working Party of the Clinical Genetics Society (UK). The genetic testing of children. J Med Genet 1994;31:785-97.

18 Gibson LE, Cooke RE. A test for concentration of electrolytes in sweat in cystic fibrosis of the pancreas utilizing pilocarpine by iontophoresis. Pediatrics 1959;23:545-9.

19 Farrell PM, Koscik RE. Sweat chloride concentrations in infants homozygous or heterozygous for F508del cystic fibrosis. Pediatrics 1996;97:524-8.

20 Di Sant'Agnese PA, Darling RC, Perera GA, et al. Abnormal electrolyte composition of sweat in cystic fibrosis of the pancreas: clinical significance and relationship to the disease. Pediatrics 1953;12:549-63.

21 Desmarquest $\mathbf{P}$, Feldman $D$, Tamalat $A$, et al. Genotype analysis and phenotypic manifestation of children with intermediate sweat chloride test results. Chest 2000;118:1591-7.

22 Lebecque P, Leal T, De Boeck K, et al. Mutations of the cystic fibrosis gene and intermediate sweat chloride levels in children. Am J Respir Crit Care Med 2002;165:757-63.

23 Boucher RC. Regulation of airway surface liquid volume by human airway epithelia. Pflugers Arch 2003;445:495-8

$24 \mathrm{http}: / /$ genet.sickkids.on.ca/cgi-bin/WebObjects/MUTATION

25 Dequeker E, Cuppens H, Dodge J, et al. Recommendations for quality improvement in genetic testing for cystic fibrosis. European Concerted Action on Cystic Fibrosis. Eur J Hum Genet 2000;8:S1-24.

26 Cuppens H, Marynen P, De Boeck K, et al. Detection of $98.5 \%$ of the mutations in 200 Belgian cystic fibrosis alleles by reverse dot blot and sequencing of the complete coding regin and exon/intron junctions of the CFTR gene. Genomics 1993; 18:693-7.

27 Le Maréchal C, Audrezet MP, Quere I, et al. Complete and rapid scanning of the cystic fibrosis conductance regulator (CFTR) gene by denaturing highperformance liquid chromatography (D-HPLC): major implications for genetic counselling. Hum Genet 2001;108:290-8.

28 Audrezet MP, Chen JM, Raguenes O, et al. Genomic rearrangements in the CFTR gene: extensive allelic heterogeneity and diverse mutational mechanisms. Hum Mutat 2004;23:343-57.

29 Chillón M, Casals T, Mercier B, et al. Mutations in the cystic fibrosis gene in patients with congenital absence of the vas deferens. NEngl J Med 1995:332: 1475-80.

30 Cuppens $\mathbf{H}$, Lin W, Jaspers $M$, et al. Polyvariant mutant cystic fibrosis transmembrane conductance regulator genes: the polymorphic (TG)m locus explains the partial penetrance of the T5 polymorphism as a disease mutation. J Clin Invest 1998;101:487-96.

31 Noone PG, Pue CA, Zhou Z, et al. Lung disease associated with the IVS8 5T allele of the CFTR gene. Am J Respir Crit Care Med 2000;162:1919-24.

32 Groman JD, Hefferon TW, Casals T, et al. Variation in a repeat sequence determines whether a common variant of the cystic fibrosis transmembrane conductance regulator gene is pathogenic or benign. Am J Hum Genet 2004;74:176-9.

33 Mekus F, Ballmann M, Bronsveld I, et al. Cystic fibrosis like disease unrelated to cystic fibrosis transmembrane conductance regulator. Hum Genet 1998; 102:582-6.

34 Donaldson SH, Boucher RC. Update on pathogenesis of cystic fibrosis lung disease. Curr Opin Pulm Med 2003;9:486-91.

35 Delmarco A, Pradal U, Cabrini G, et al. Nasal potential difference in cystic fibrosis patients presenting borderline sweat test. Eur Respir $J$ 1997; 10:1145-9.

36 Hubert D, Jajac I, Bienvenu T, et al. Diagnosis of cystic fibrosis in adults with diffuse bronchiectasis. J Cyst Fibros 2004;3:15-22.

37 Hofmann T, Bohmer O, Huls G, et al. Conventional and modified nasal potential difference measurement in cystic fibrosis. Am J Respir Crit Care Med 1997; 155:1908-13.

38 Gelrud A, Sheth S, Baneriee S, et al. Analysis of CFTR function in patients with pancreas divisum and recurrent acute pancreatitis. Am J Gastroenterol 2004;99:1557-62

39 Schuler D, Sermet-Gaudelus I, Wilschanski M, et al. Basic protocol for transepithelial potential difference measurements. J Cyst Fibros 2004;3:151-6.

40 Standaert TA, Boitano L, Emerson J, et al. Standardized procedure for measurement of nasal potential difference: an outcome measure in multicenter cystic fibrosis trials. Pediatr Pulmonol 2004;37:385-92.

41 Wilschanski M, Famini $\mathrm{H}$, Strauss-Liviatan N, et al. Nasal potential difference measurements in patients with atypical cystic fibrosis. Eur Respir J 2001;17:1208-15

42 Hallberg K, Reims A, Strandvik B. Electrogenic ion transport in duodenum, an aid in cystic fibrosis diagnosis. Scand J Gastroenterol 2000;35:1106-9.

43 Veeze HJ, Sinaasappel M, Bijman J, et al. lon transport abnormalities in rectal suction biopsies from children with cystic fibrosis. Gastroenterology 1991; 101:398-403.

44 De Jonge HR, Ballmann $M$, Veeze $H$, et al. Ex vivo $C F$ diagnosis by intestinal current measurements (ICM) in small aperture, circulating Ussing chambers. J Cyst Fibros 2004;3:159-63.

45 Mall M, Hirtz S, Gonska T, et al. Assessment of CFTR function in rectal biopsies for the diagnosis of cystic fibrosis. J Cyst Fibros 2004:3:165-9.

46 Taylor CJ, Baxter PS, Hardcastle J, et al. Failure to induce secretion in jejunal biopsy samples from children with cystic fibrosis. Gut 1988;29:957-62.

47 Hug MJ, Tummler B. Ex vivo CF diagnosis by intestinal current measurement (ICM) in small aperture, circulating Ussing chambers. J Cyst Fibros 2004;3(Suppl 2):157-8 